\title{
Diastereoselective synthesis and molecular docking studies of novel fused tetrahydropyridine derivatives as new inhibitors of HIV protease
}

\author{
Ali A. Mohammadi, ${ }^{a} *$ Salman Taheri, ${ }^{a}$ Ali Amouzegar, ${ }^{a}$ Reza Ahdenov, ${ }^{a}$ Mohammad Reza Halvagar, ${ }^{\text {a }}$ Ahmad Shahir \\ Sadr, \\ ${ }^{a}$ Chemistry and Chemical Engineering Research Center of Iran (CCERCI), PO Box 14335-186, Tehran, I.R. Iran \\ ${ }^{b}$ Bioinformatics Research Center, Sabzevar University of Medical Sciences, Sabzevar, Iran \\ ${ }^{b}$ Bioinformatics Research Center,Cheragh Medical institute \&Hospital, Kabul, Afghanistan
}

\begin{abstract}
An efficient one-pot, catalyst-free, and four-components procedure for the synthesis of novel 10b-hydroxy-4-nitro-5-phenyl-2,3,5,5a-tetrahydro-1 $H$-imidazo[1,2-a]indeno[2,1-e]pyridin-6(10bH)one derivatives from corresponding diamine, nitro ketene dithioacetal, aldehydes and 1,3-indandione in ethanol has been achieved upon a Knoevenagel condensation-Michael addition-tautomerismcyclisation sequence. All the newly synthesized compounds were screened for molecular docking studies. Molecular docking studies were carried out using the crystal structure of HIV protease enzyme. Some of the compounds obtain minimum binding energy and good affinity toward the active pocket of HIV protease enzyme in compare with Saquinavir as a standard HIV protease inhibitor.
\end{abstract}

Keywords: Four-component, Ketene dithioacetal, Tetrahydropyridine, Molecular docking, HIV protease inhibitor

\section{Introduction}

Nitrogen heterocyclic compounds such as tetrahydropyridine are important class of organic compounds. They have been reported as starting materials for total synthesis of the natural compounds such as Reserpine,[1] Catharanthine,[2] Lepadin B.[3] They are also well known for their hypertension, anti-cancer activity (e.g., vinblastine), and cancer metastasis. In addition, the tetrahydropyridine ring system is a widely distributed structural framework that is involved in a number of pharmaceuticals

* Corresponding author. Tel.: +98-21-44787798; fax: +98-21-44788454; e-mail: aliamohammadi@ccerci.ac.ir 
and natural products,[4],[5] such as, Haouamine A, and B, isolated from the ascidian Aplidium haourianum,[6] (-)-grandisine G, isolated from an Australian rainforest tree Elaeocarpus grandis, which was found to show potent activity in the human $\sigma$-opioid receptor binding affinity,[7] and Lysergic acid.[8] Furthermore, during the last decade, such compounds have shown interesting pharmacological properties like anti-inflammatory,[9] antimycobacterial,[10] antimalarials,[11] dopamine D1 receptor,[12] GABA,[13] and CB1 receptors.[14] (Fig. 1)

In recent years, due to some specific interest in the biological activity of tetrahydropyridine, several synthetic procedures have been reported for these compounds,[15-18] but the preparation of corresponding tetrahydropyridine analogues have not yet been evolved.

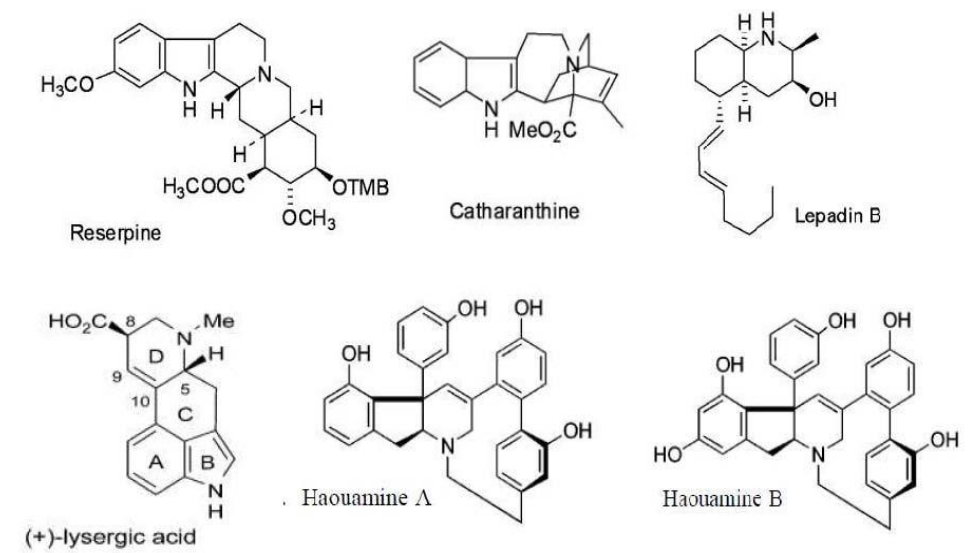

Fig 1. Examples of tetrahydropyridin derivatives as natural products and pharmaceuticals and biological active agents

Multicomponent condensations (MCCs) constitute a resourceful synthetic strategy for rapid and efficient library generation due to the fact that the products are formed in a single step and the diversity can be achieved simply by varying the reacting components. Recently, we have reported the preparation of bioactive nitrogen-containing heterocyles[19, 20] such as imidazoles,[21] quinazolines,[22] cis-isoquinolonic acids,[23] spirooxindoles,[24] dihyroquinazolinones,[25] via multicomponent reactions. In the context of our interest in exploring a convenient access to 
heterocyclic systems, we wish to report herein our result on a four components diastereoselective synthesis of 10b-hydroxy-4-nitro-5-phenyl-2,3,5,5a-tetrahydro-1H-imidazo[1,2-a]indeno[2,1-e]pyridin$6(10 \mathrm{bH})$-one 5a-i in the absence of organic or inorganic catalyst and in an one-pot procedure. (Scheme1)

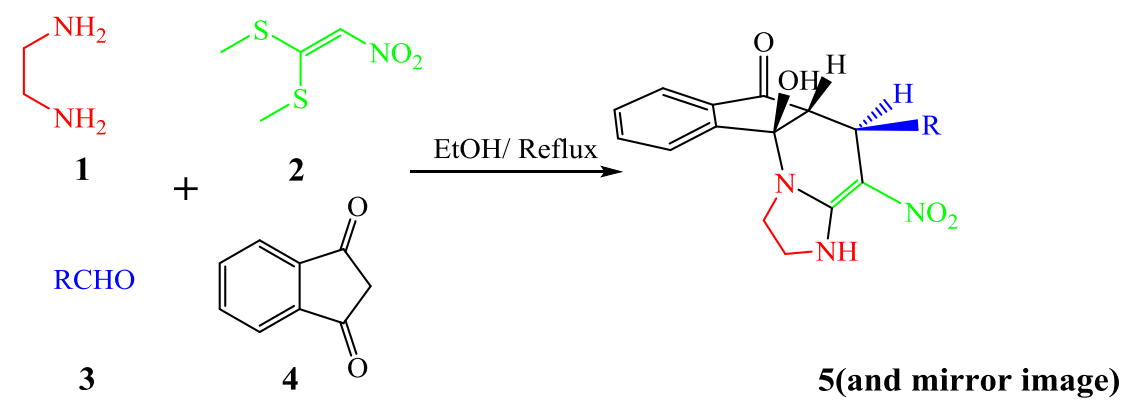

Scheme 1. Diastereoselective synthesis of 10b-hydroxy-4-nitro-5-phenyl2,3,5,5a-tetrahydro-1 $H$ - imidazo[1,2-a]indeno[2,1-e]pyridin-6(10bH)-one $\mathbf{5}$.

\section{Results and Discussion}

\subsection{Chemistry}

The reaction condition has been optimized by the reaction of ethylene diamine $\mathbf{1}$ (1mmol), 1,1bis(methylthio)-2-nitro ethylene 2 (1 $\mathrm{mmol})$, benzaldehyde $\mathbf{3 a}(1 \mathrm{mmol})$, and indandione $\mathbf{4}$ (1 $\mathrm{mmol})$ to result in, 10b-hydroxy-4-nitro-5-phenyl-2,3,5,5a-tetrahydro -1H-imidazo[1,2-a] indeno[2,1-e]pyridin$6(10 \mathrm{bH})$-one 5a. The reaction mixture was refluxed for $150 \mathrm{~min}$ until the indandione disappeared, monitored by TLC analysis to yield $96 \%$ of the product.

The effect of various solvents $\left(\mathrm{CH}_{3} \mathrm{CN}\right.$, toluene, THF, $\mathrm{CH}_{2} \mathrm{Cl}_{2}, \mathrm{H}_{2} \mathrm{O}$, and EtOH) and temperatures (rt, $30{ }^{\circ} \mathrm{C}, 50{ }^{\circ} \mathrm{C}, 60{ }^{\circ} \mathrm{C}$, and reflux) were established. These results indicate that the ethanol solvent at reflux condition provides the highest yield and the shortest reaction time.

To generalize this reaction procedure, we explored the scope of this reaction by varying the structure of aldehyde 3 derivatives with indandione, ethylene diamine and 1,1-bis(methylthio)-2-nitro ethylene. The results are listed in Table 1.

All products are new racemic compounds, owned to our polari metric observation, and their structures were deduced by a single crystal X-ray crystallography, IR, ${ }^{1} \mathrm{H}$ NMR and ${ }^{13} \mathrm{C}$ NMR Spectra. The mass 
spectra of these compounds displayed molecular ions at the appropriate mlz values (See the SI and the experimental section).

The ${ }^{1} \mathrm{HNMR}$ spectrum shows two singlets close to 3.5-3.7 ppm and 4.9-5.3 ppm that were attributed to the $\mathrm{H}_{10}$ and $\mathrm{H}_{11}$ hydrogen atoms (Fig. 2, atom labeling) in all of the racemic cycloadducts 5 respectively. This is so because of the close proximity of these two hydrogen atoms with a torsion angle (H10-C4-C12-H11 83.5(2), Fig. 2) of less than 90 (according to Karla's equation)[26] (Fig. 2).

The ${ }^{1}$ HNMR spectrum also shows two singlets at $\delta=6.04-7.19$ and 9.04-9.21 ppm which are attributed to the $\mathrm{NH}$ an $\mathrm{OH}$ groups. The stretching frequency of the same groups appears at 3295-3350 and 3055$3195 \mathrm{~cm}^{-1}$ in the IR spectrum.

According to the results, a plausible mechanism for the formation of product is shown in scheme 2 . Initially, condensation of ethylene diamine 1 with 1,1-bis(methylthio)-2-nitro ethylene 2, and aldehyde 3 with indandione 4 resulted in the ketene diaminal 6 and compound 7 via Knoevenagel condensation. Then, the condensation compound 7 with keten diaminal 6, gives intermediate $\mathbf{8}$. Subsequently, this intermediate undergoes a [1,3] hydrogen shift to give intermediate $\mathbf{9}$. Once intermediate $\mathbf{9}$ is formed, a nucleophilic attack takes place by the nitrogen group in conformity with Baldwin's rules (6-Exotrig) yield the final products. (Scheme 2)

Notably, among all of the possible diastereoisomers that can form in this reaction, we have found that this reaction is highly stereoselective in the preparation of racemic product of $(5 \mathrm{~S}, 5 \mathrm{aR}, 10 \mathrm{bR})$ and (5R,5aS, 10bS)10b-hydroxy-4-nitro-5-phenyl-2,3,5,5a-tetrahydro-1H-imidazo[1,2-a]indeno[2,1-e] pyridin-6(10bH)-one 5. The constitution of 5e was clarified by single crystal X-ray crystallography.[27] (Figure 2)

Encouraged by this success, we extended our investigations on in silico docking studies of this new synthesized compounds utilizing Autodock Vina[28] and Autodock 4[29] programs. 
We have performed a chemical structure search of all new synthesized compounds at the NCBI PubChem (patents) database to retrieve the related compounds and analogues. The SMILES encoding search parameters were set at $85 \%$ similarity subjected. (Table 2)

Interestingly, among the results of the similarity search for all of the new synthesized compounds on the drug database there was Saquinavir drug that is well-known HIV protease enzyme.[30]

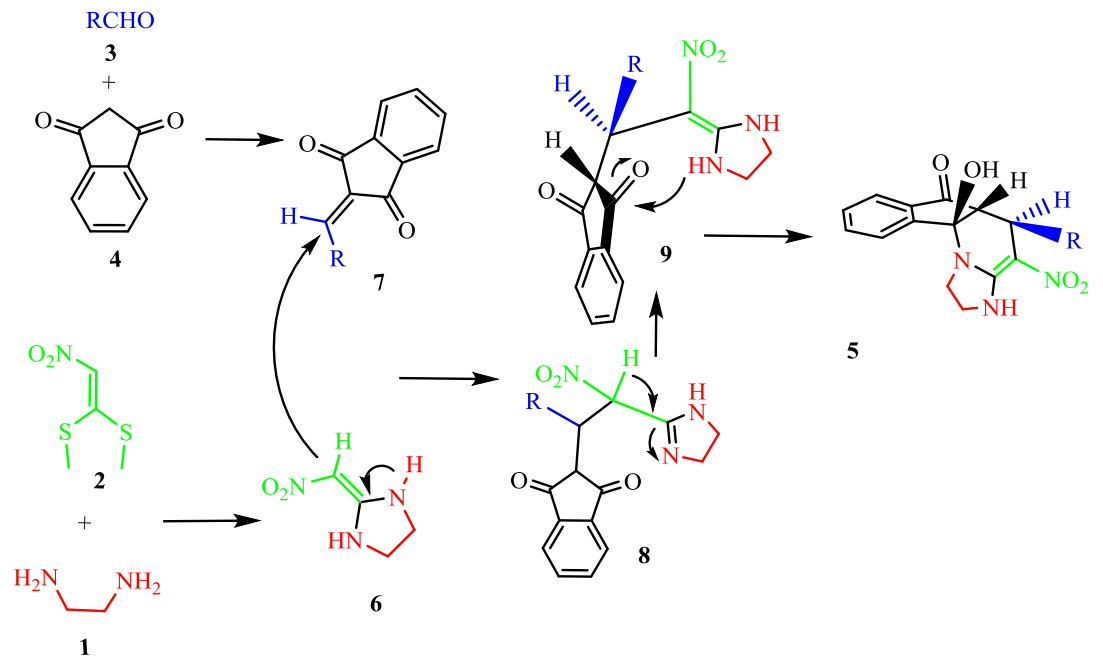

Scheme 2. Proposed mechanism for the formation of products $\mathbf{5 a - i}$

Table 1: Catalyst free synthesis of 10b-hydroxy-4-nitro-5-phenyl-2,3,5,5a-tetrahydro- $1 H$ imidazo[1,2-a]indeno [2,1-e] pyridin-6 $(10 \mathrm{~b} H)$-one 5

\begin{tabular}{cllll}
\hline Products & $\mathrm{R}$ & Time $(\mathrm{min})$ & Yield $(\%)^{\mathrm{a}}$ & $\mathrm{Mp}\left({ }^{\circ} \mathrm{C}\right)$ \\
\hline $\mathbf{5 a}$ & $\mathrm{Ph}$ & 150 & 85 & $223-224$ \\
$\mathbf{5 b}$ & $4-\mathrm{MeC}_{6} \mathrm{H}_{4}$ & 180 & 81 & $201-203$ \\
$\mathbf{5 c}$ & $4-$ pyridine & 120 & 88 & $222-224$ \\
$\mathbf{5 d}$ & $3-\mathrm{ClC}_{6} \mathrm{H}_{4}$ & 120 & 89 & $218-220$ \\
$\mathbf{5 e}$ & $2-\mathrm{NO}_{2} \mathrm{C}_{6} \mathrm{H}_{4}$ & 70 & 91 & $213-215$ \\
$\mathbf{5 f}$ & $3-\mathrm{NO}_{2} \mathrm{C}_{6} \mathrm{H}_{4}$ & 60 & 97 & $217-218$ \\
$\mathbf{5 g}$ & $4-\mathrm{BrC}_{6} \mathrm{H}_{4}$ & 120 & 90 & $212-213$ \\
$\mathbf{5 h}$ & $4-\mathrm{NO}_{2} \mathrm{C}_{6} \mathrm{H}_{4}$ & 60 & 96 & $206-207$ \\
$\mathbf{5 i}$ & 3-MeOC $6 \mathrm{H}_{4}$ & 180 & 81 & $210-212$ \\
\hline \multicolumn{5}{c}{ a } \\
\end{tabular}




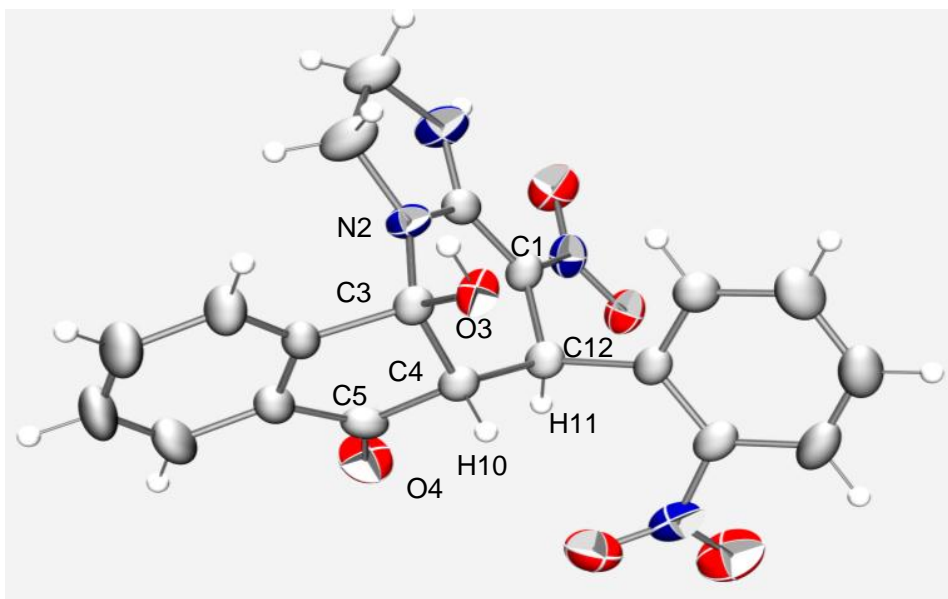

Figure 2. Crystal structure of the formula unit of 5e. Solvent molecule (THF) is omitted for clarity (thermal ellipsoids set at the $40 \%$ probability level). Selected bond lengths [ $[\AA]$ and angles [ [ ${ }^{\circ}$ : C3-O3 1.400(3), C3-C4 1.545(4), C4-C5 1.528(4), C3N2 1.463(4), C5-O4 1.205(4), C12-C1 1.498(4), O4-C5-C4 125.2(3), C4-C3-O3 109.8(2), N2-C3-C4 109.1(2), C1-C12-C4 110.0(3), O3-C3-N2 111.3(3), H10-C4-C12-H11 83.5(2).

\subsection{Molecular Docking}

Considering HIV protease enzyme as the target receptor, comparative and automated docking studies with newly synthesized candidate lead compounds and Saquinavir where performed to determine the best in silico conformation. The native crystal structure of HIV protease enzyme in complex with Saquinavir was obtained from Protein Data Bank (http://www.pdb.org/pdb/home/ home .do) with the PDB ID: 1HXB which was resolved at $2.30 \mathrm{~A}^{\circ}$ using X-ray[31] crystallography method. Saquinavir was obtained from Pubchem (http://pubchem. ncbi. nlm. nih.gov/) database ID: 60787 shows in Fig. 3.

Fig. 4 shows the results of the docking analyses depicting the best, stable conformations and binding site of all the synthesized molecules and the standard drug (Saquinavir) by using the program Autodock 4. According to this results all of these compounds exhibit hydrogen bonding with one or more amino acids of HIV protease receptor similar to the standard drug. (Fig. 5) In the case of the Saquinavir the molecular structure that was obtained from Pubchem database compared with the protein bonded Saquinavir which was obtained from Protein Data Bank. In both cases three H-bonding site are engaged in the receptor cavity namely: GLY27.A, ILE50.B, \& VAL82.A (Pubchem) and GLY48.B, ASP25.A, \& ASP29.B amino acids (Protein Data Bank) (Fig. 5). The final docking score was 
calculated in terms of Kcal/mole for each docking experiment. Interactions with the active site of target proteins are given in Table 3.

The result has shown a good docking score for the tested compounds (5a-i) ranging from -8.8 to -9.9 (Auto Dock Vina) and -7.73 to $-9.24 \mathrm{Kcal} / \mathrm{mol}$ (Auto Dock 4) and for the tested compounds (mirror image 5a-i) ranging from -8.0 to -9.8 (Auto Dock Vina) and -7.58 to $-8.23 \mathrm{Kcal} / \mathrm{mol}$ (Auto Dock 4). The standard drug compound, Saquinavir, gave a docking score of -9.7 (Auto Dock Vina), and -12.63 (Auto Dock 4) for the structure extracted from Pubchem database and -10.7 (Auto Dock Vina), and -12.20 (Auto Dock 4) Kcal/mol for the crystal structure extracted from the protein structure Databank (PDB). Interestingly, although the molecular size of the newly synthesized compounds are very smaller to Saquinavir but according to our in silico studies some of the newly synthesized derivatives reveal good binding energy toward the target protein.

It was observed that the compound containing $\mathrm{NO}_{2}$ group in the meta position of the phenyl ring, 10bhydroxy-4-nitro-5-phenyl-2,3,5,5a-tetrahydro- $1 H$-imidazo[1,2-a]indeno[2,1-e]pyridin-6(10b $H)$-one $5 \mathbf{5 f}$ shows a better binding energy $(-9.24 \mathrm{kcal} / \mathrm{mol})$ compare to the other derivatives when the results obtained from Autodock 4. The results are more promising when the in silico docking studies was carried out by Autodock Vina program. In this case the non- derivatized phenyl group 5a shows a better binding energy $(-9.9 \mathrm{kcal} / \mathrm{mol})$ than the other compounds and the binding energy of Saquinavir extracted from the Pubchem database. (Table 3)

\section{Conclusion}

In summary, we have described an efficient strategy, and convenient green synthesis for the preparation of novel tetrahydropyridine in a four components reaction including ethylene diamine, 1,1bis(methylthio)2-nitro ethylene, benzaldehyde, and indandione, in the absence of catalysts. The method offers several advantages including high yield of products, and easy experimental workup procedure. Surprisingly, this reaction is diastereoselective in the preparation of racemic $(5 \mathrm{~S}, 5 \mathrm{aR}, 10 \mathrm{bR})$ and (5R,5aS,10bS)-10b-hydroxy-4-nitro-5-phenyl-2,3,5,5a-tetrahydro-1H-imidazo[1,2-a]indeno [2,1-e] 
pyridine-6(10bH)-one, which makes it a useful procedure for the synthesis of these compounds. Furthermore, molecular docking study indicated that all of the herein reported compounds could snugly occupy the active site of HIV protease enzyme, which could make them a plausible candidate for the target site. The in silico molecular docking study results showed that, some of these compounds exhibit minimum binding energy and good affinity toward the active pocket of HIV protease enzyme in compare with Saquinavir as a standard HIV protease inhibitor, thus, could make them a presentable candidate for a future investigation on HIV protease inhibitors.

\section{Experimental}

\section{Material And Measurements}

Melting points were obtained in open capillary tubes and were measured on an electrothermal 9200 apparatus. Mass spectra were recorded on a Shimadzu QP 1100 BX mass spectrometer. The IR spectra were recorded on $\mathrm{KBr}$ pellets on a Shimadzu IR470 spectrophotometer. ${ }^{1} \mathrm{H}$ and ${ }^{13} \mathrm{C}$ NMR spectra were determined on a Bruker 300 DRX Avance instrument at 300 and $75 \mathrm{MHz}$. Elemental analysis for C, H and $\mathrm{N}$ were performed using a Heraus CHN rapid analyzer. Single crystal data were collected on diffractometer equipped with a STOE IPDS/2T imaging plate detector, T =298(2) K, MoKa radiation $\left(\lambda \mathrm{MoK}_{\alpha}=0.71073 \AA\right)$, graphite monochromator, Structure solution by direct methods, full-matrix-leastsquares refinement against $\mathrm{F} .{ }^{1} \mathrm{H}$ and ${ }^{13} \mathrm{C}$ chemical shifts calibrated with TMS (tetramethylsilane).

\section{General experimental procedure for synthesis of 10b-hydroxy-4-nitro-5-phenyl-}

\section{2,3,5,5atetrahydro-1H-imidazo[1,2-a]indeno[2,1-e]pyridin-6(10bH)-one derivatives $\quad 5 a-i: \quad$ A} mixture of ethylene diamine 1 ( $1 \mathrm{mmol}, 0.066 \mathrm{ml})$, 1,1-bis(methylthio)-2-nitro ethylene 2 (1 mmol, $0.165 \mathrm{~g}$ ) and $10 \mathrm{ml} \mathrm{EtOH}$ in a $50 \mathrm{ml}$ flask was stirred at reflux for $6 \mathrm{~h}$. After completion of the reaction (monitored by TLC, ethylacetate/n-hexane, 2:1), benzaldehyde 3a (1 mmol, $0.1 \mathrm{ml}$ ) and indandione 4 ( $1 \mathrm{mmol}, 0.146 \mathrm{~g})$, was added to the reaction mixture and was stirred at reflux for the time period as indicated in table 1 . The reaction mixture was allowed to cool down to room temperature and the resulting solid was separated by filtration. The crude product was washed with hot ethanol to afford the 
purified product.

\section{0b-Hydroxy-4-nitro-5-phenyl-2,3,5,5a-tetrahydro-1 $H$-imidazo[1,2-a]indeno[2,1-e]pyridin-6}

(10bH)-one (5a): White solid, MP: 223-224 C, IR (KBr): $3357(\mathrm{NH}), 3140(\mathrm{OH}), 2904,1725(\mathrm{C}=\mathrm{O})$, $1647 \mathrm{~cm}^{-1}$; ${ }^{1} \mathrm{H}$ NMR (300 MHz, DMSO-d6) $\delta_{\mathrm{H}:} 3.20-3.29\left(\mathrm{~m}, 1 \mathrm{H}, \mathrm{CH}_{2}\right), 3.39-3.53\left(\mathrm{~m}, 1 \mathrm{H}, \mathrm{CH}_{2}\right), 3.57$ (s. 1H, CH), 3.64-3.74 (m, 1H, $\left.\mathrm{CH}_{2}\right), 3.96-4.05\left(\mathrm{~m}, 1 \mathrm{H}, \mathrm{CH}_{2}\right), 4.94(\mathrm{~s}, 1 \mathrm{H}, \mathrm{CH}), 6.94$ (s, 1H, OH), 7.19 (t, 1H, J=6.6 Hz, Ar-H), 7.26-7.34 (m, 4H, Ar-H), 7.62-7.66 (m, 1H, Ar-H), 7.60 (d, 1H, J=7.5 Hz, Ar$\mathrm{H}), 7.82-7.87(\mathrm{~m}, 2 \mathrm{H}, \mathrm{Ar}-\mathrm{H}), 9.08(\mathrm{~s}, 1 \mathrm{H}, \mathrm{NH}) \mathrm{ppm} ;{ }^{13} \mathrm{C} \mathrm{NMR}(75 \mathrm{MHz}, \mathrm{DMSO}-d 6) \delta_{\mathrm{c}:} 36.8,42.3$, $43.5,63.5,85.8,104.2,123.5,124.8,126.3,127.7,128.2,130.5,133.0,135.1,142.8,151.2,154.3$, 198.6 ppm. MS (EI, 70eV) m/z: $363\left(\mathrm{M}+\right.$ ). Anal.Calcd for $\mathrm{C}_{20} \mathrm{H}_{17} \mathrm{~N}_{3} \mathrm{O}_{4}: \mathrm{C}, 66.11 ; \mathrm{H}, 4.72 ; \mathrm{N}, 11.56 \%$. Found: C, 66.00; H, 4.61; N, 11.44\%.

\section{0b-Hydroxy-4-nitro-5-p-tolyl-2,3,5,5a-tetrahydro-1H-imidazo[1,2-a]indeno[2,1-e]pyridin-6}

(10bH)-one (5b): White solid, MP: 201-203 C, IR (KBr): $3352(\mathrm{NH}), 3151(\mathrm{OH}), 2898,1721(\mathrm{C}=\mathrm{O})$, $1594 \mathrm{~cm}^{-1} ;{ }^{1} \mathrm{H}$ NMR (300 MHz, DMSO-d6) $\delta_{\mathrm{H}}: 2.26$ (s, 3H, $\left.\mathrm{CH}_{3}\right)$ 3.20-3.28 (m, 1H, $\left.\mathrm{CH}_{2}\right), 3.47-3.52$ (m, 2H, $\left.\mathrm{CH}_{2}, \mathrm{CH}\right), 3.66-3.72\left(\mathrm{~m}, 1 \mathrm{H}, \mathrm{CH}_{2}\right), 3.97-4.04\left(\mathrm{~m}, 1 \mathrm{H}, \mathrm{CH}_{2}\right), 4.90(\mathrm{~s}, 1 \mathrm{H}, \mathrm{CH}), 6.90(\mathrm{~s}, 1 \mathrm{H}$, $\mathrm{OH}), 7.07$ (d, 2H, J=7.5 Hz, Ar-H), 7.19 (d, 2H, J=7.8 Hz, Ar-H), 7.64-7.66 (m, 1H, Ar-H), 7.75 (d, $1 \mathrm{H}, J=7.5 \mathrm{~Hz}, \mathrm{Ar}-\mathrm{H}), 7.84-786$ (m, 2H, Ar-H), 9.06 (s, 1H, NH) ppm; ${ }^{13} \mathrm{C}$ NMR (100 MHz, DMSO-d6) $\delta \mathrm{C}: 21.0,36.7,42.6,43.8,63.9,86.1,104.6,123.8,125.2,127.9,129.1,130.8,133.4,135.4,135.5$, 140.2, 151.6, 154.6, 198.9 ppm. MS (EI, 70eV) m/z: $337(\mathrm{M}+)$. Anal.Calcd for $\mathrm{C}_{21} \mathrm{H}_{19} \mathrm{~N}_{3} \mathrm{O}_{4}: \mathrm{C}, 66.83$; H, 5.07; N, $11.13 \%$. Found: C, 66.74; H, 4.96; N, 11.12\%.

\section{0b-Hydroxy-4-nitro-5-(pyridin-4-yl)-2,3,5,5a-tetrahydro-1H-imidazo[1,2-a]indeno[2,1-e]}

pyridin-6(10bH)- one (5c): White solid, MP: 222-224ํㅡ, IR (KBr): 3318 (NH), 3055 (OH), 2906, $1729(\mathrm{C}=\mathrm{O}), 1597 \mathrm{~cm}^{-1} ;{ }^{1} \mathrm{H}$ NMR (400 MHz, DMSO-d6) $\delta \mathrm{H}: 3.18-3.25\left(\mathrm{~m}, 1 \mathrm{H}, \mathrm{CH}_{2}\right), 3.45-3.55(\mathrm{~m}$, $\left.1 \mathrm{H}, \mathrm{CH}_{2}\right), 3.62(\mathrm{~s}, 1 \mathrm{H}, \mathrm{CH}), 3.66-3.73\left(\mathrm{~m}, 1 \mathrm{H}, \mathrm{CH}_{2}\right), 3.96-4.29\left(\mathrm{~m}, 1 \mathrm{H}, \mathrm{CH}_{2}\right), 4.93(\mathrm{~s}, 1 \mathrm{H}, \mathrm{CH}), 7.04(\mathrm{~s}$, 1H, OH); 7.33 (d, 2H, J=5.6 Hz, Ar-H), 7.63-7.66 (m, 1H, Ar-H), 7.77 (d, 1H, J=7.6 Hz, Ar-H), 7.837.89 (m, 2H, Ar-H), 8.47 (d, 2H, J=5.6 Hz, Ar-H), 9.04 (s, 1H,NH) ppm; ${ }^{13} \mathrm{C}$ NMR (100 MHz, DMSO- 
d6) ¿c: $36.6,42.7,43.8,63.1,85.9,103.1,123.4,123.9,125.1,130.9,133.3,135.5,149.8,151.3,152.0$, 154.5, 198.2 ppm. MS (EI, 70eV) m/z: $364\left(\mathrm{M}^{+}\right)$. Anal.Calcd for $\mathrm{C}_{19} \mathrm{H}_{16} \mathrm{~N}_{4} \mathrm{O}_{4}: \mathrm{C}, 62.63 ; \mathrm{H}, 4.43 ; \mathrm{N}$, $15.38 \%$. Found: C, 62.52; H, 4.33; N, 15.26\%.

\section{5-(3-Chlorophenyl)-10b-hydroxy-4-nitro-2,3,5,5a-tetrahydro-1H-imidazo[1,2-a]indeno[2,1-e]}

pyridin-6(10bH)-one (5d): White solid, MP: 218-220 C, IR (KBr): 3325 (NH), $3110(\mathrm{OH}), 2909$, $1723(\mathrm{C}=\mathrm{O}), 1599 \mathrm{~cm}^{-1} ;{ }^{1} \mathrm{H}$ NMR $(300 \mathrm{MHz}, \mathrm{DMSO}-d 6) \delta_{\mathrm{H}}: 3.25-3.28\left(\mathrm{~m}, 1 \mathrm{H}, \mathrm{CH}_{2}\right), 3.42-3.46(\mathrm{~m}$, $\left.1 \mathrm{H}, \mathrm{CH}_{2}\right), 3.51-3.58\left(\mathrm{~m}, 2 \mathrm{H}, \mathrm{CH}_{2}, \mathrm{CH}\right), 3.63-3.75\left(\mathrm{~m}, 1 \mathrm{H}, \mathrm{CH}_{2}\right), 4.91(\mathrm{~s}, 1 \mathrm{H}, \mathrm{CH}), 7.17(\mathrm{~s}, 1 \mathrm{H}, \mathrm{OH})$, 7.24-7.62 (m, 4H, Ar-H), 7.72-7.83 (m, 4H, Ar-H), 9.08 (s, 1H, NH) ppm; ${ }^{13} \mathrm{CNMR}$ (75 MHz, DMSOd6) cc: $37.0,42.8,44.1,63.8,86.2,86.5,105.1,105.2,124.1,125.4,128.1,131.3,133.3,133.6,135.6$, 135.9, 140.8, 151.5, 155.0, 199.6 ppm. MS (EI, 70eV) m/z: $397(\mathrm{M}+)$. Anal.Calcd for $\mathrm{C}_{20} \mathrm{H}_{16} \mathrm{ClN}_{3} \mathrm{O}_{4}$ : C, 60.38; H, 4.05; N, $10.56 \%$. Found: C, 60.26; H, 3.06; N, 10.45\%.

\section{0b-Hydroxy-4-nitro-5-(2-nitrophenyl)-2,3,5,5a-tetrahydro-1H-imidazo[1,2-a]indeno[2,1-e]}

pyridin-6(10bH)-one (5e): White solid, MP: 213-215ㄷ, IR (KBr): 3348 (NH), $3346(\mathrm{OH}), 2917$, $1729(\mathrm{C}=\mathrm{O}), 1599 \mathrm{~cm}^{-1} ;{ }^{1} \mathrm{H}$ NMR $(300 \mathrm{MHz}, \mathrm{DMSO}-d 6) \delta_{\mathrm{H}}: 3.17-3.26\left(\mathrm{~m}, 1 \mathrm{H}, \mathrm{CH}_{2}\right), 3.51-3.57(\mathrm{~m}$, $\left.1 \mathrm{H}, \mathrm{CH}_{2}\right), 3.65(\mathrm{~s}, 1 \mathrm{H}, \mathrm{CH}), 3.71-3.77(\mathrm{~m}, 1 \mathrm{H}, \mathrm{CH}), 3.98-4.07\left(\mathrm{~m}, 1 \mathrm{H}, \mathrm{CH}_{2}\right), 5.31(\mathrm{~s}, 1 \mathrm{H}, \mathrm{CH}), 7.11(\mathrm{~s}$, 1H, OH), 7.47 (t, 1H, J=7.5 Hz, Ar-H), 7.53 (d, 1H, J=7.8 Hz, Ar-H), 7.62-7.67 (m, 2H, Ar-H), 7.787.93 (m, 4H,Ar-H), 9.12 (s, 1H, NH) ppm; ${ }^{13} \mathrm{C}$ NMR (75 MHz, DMSO-d6) $\delta_{\mathrm{c}}: 32.5,42.3,43.4,56.0$, $61.5,85.3,103.6,123.5,123.9,124.5,127.8,129.9,130.4,132.8,135.0,137.0,149.3,150.9,154.2$, 196.8 ppm. MS (EI, 70eV) m/z: $408(\mathrm{M}+)$. Anal.Calcd for $\mathrm{C}_{20} \mathrm{H}_{16} \mathrm{~N}_{4} \mathrm{O}_{6}: \mathrm{C}, 58.82 ; \mathrm{H}, 3.95 ; \mathrm{N}, 13.72 \%$. Found: C, 58.71; H, 3.83; N, 13.62\%.

\section{0b-hydroxy-4-nitro-5-(3-nitrophenyl)-2,3,5,5a-tetrahydro-1H-imidazo[1,2-a]indeno[2,1-}

e]pyridin-6(10bH)-one (5f): White solid, MP: 217-218 C, IR (KBr): 3333 (NH), 3124 (OH), 2900, $1727(\mathrm{C}=\mathrm{O}), 1585 \mathrm{~cm}^{-1} ;{ }^{1} \mathrm{H}$ NMR (300 MHz, DMSO-d6) $\delta_{\mathrm{H}:}$ 3.17-3.26 (m, 1H, CH 2$), 3.47-3.57(\mathrm{~m}$, $\left.1 \mathrm{H}, \mathrm{CH}_{2}\right), 3.69-3.76\left(\mathrm{~m}, 2 \mathrm{H}, \mathrm{CH}_{2}, \mathrm{CH}\right), 3.97-4.07\left(\mathrm{~m}, 1 \mathrm{H}, \mathrm{CH}_{2}\right), 5.05(\mathrm{~s}, 1 \mathrm{H}, \mathrm{CH}), 7.19(\mathrm{~s}, 1 \mathrm{H}, \mathrm{OH})$, 7.56-7.67 (m, 2H, Ar-H), 7.77 (d, 1H, J=7.8 Hz, Ar-H), 7.84-7.91 (m, 3H, Ar-H), 7.08 (d, 1H, J=8.1 
$\mathrm{Hz}, \mathrm{Ar}-\mathrm{H}), 8.20$ (s, 1H, Ar-H), 9.15 (s, 1H, NH) ppm; ${ }^{13} \mathrm{C}$ NMR (75 MHz, DMSO-d6) $\delta \mathrm{c:} \mathrm{36.5,} \mathrm{42.3,}$ $43.4,62.7,85.4,103.3,121.5,122.3,123.5,124.6,129.6,130.5,133.0,134.8,135.0,145.1,147.7$, 150.8, 154.0, 197.6 ppm. MS (EI, 70eV) m/z: $408\left(\mathrm{M}^{+}\right)$. Anal.Calcd for $\mathrm{C}_{20} \mathrm{H}_{16} \mathrm{~N}_{4} \mathrm{O}_{6}$ : C, 58.82; H, 3.95; N, $13.72 \%$. Found: C, 58.73; H, 3.84; N, $13.60 \%$.

\section{5-(4-bromophenyl)-10b-hydroxy-4-nitro-2,3,5,5a-tetrahydro-1H-imidazo[1,2-a]indeno[2,1-}

e]pyridin-6(10bH)-one (5g): White solid, MP: 212-213ํㅡ, IR (KBr) 3321 (NH), 3145 (OH), 2902, $1722(\mathrm{C}=\mathrm{O}), 1599 \mathrm{~cm}^{-1} ;{ }^{1} \mathrm{H}$ NMR $(300 \mathrm{MHz}, \mathrm{DMSO}-d 6) \delta \mathrm{H}: 3.17-3.26\left(\mathrm{~m}, 1 \mathrm{H}, \mathrm{CH}_{2}\right), 3.49-3.53(\mathrm{~m}$, 2H, $\left.\mathrm{CH}_{2}, \mathrm{CH}\right), 3.63-3.72\left(\mathrm{~m}, 1 \mathrm{H}, \mathrm{CH}_{2}\right), 3.94-4.03\left(\mathrm{~m}, 1 \mathrm{H}, \mathrm{CH}_{2}\right), 4.90(\mathrm{~s}, 1 \mathrm{H}, \mathrm{CH}), 7.03(\mathrm{~s}, 1 \mathrm{H}, \mathrm{OH})$; $7.27(\mathrm{~d}, 2 \mathrm{H}, J=8.1 \mathrm{~Hz}, \operatorname{Ar}-\mathrm{H}), 7.46(\mathrm{~d}, 2 \mathrm{H}, J=8.1 \mathrm{~Hz}, \mathrm{Ar}-\mathrm{H}), 7.61-7.65$ (m, 1H, Ar-H), 7.75 (d, $1 \mathrm{H}$, $J=7.5 \mathrm{~Hz}, \mathrm{Ar}-\mathrm{H}), 7.81-7.89(\mathrm{~m}, 2 \mathrm{H}, \mathrm{Ar}-\mathrm{H}), 9.12(\mathrm{~s}, 1 \mathrm{H}, \mathrm{NH}) \mathrm{ppm} ;{ }^{13} \mathrm{C}$ NMR $(75 \mathrm{MHz}, \mathrm{DMSO}-d 6) \delta_{\mathrm{C}}:$ $36.1,42.1,43.4,63.2,85.5,103.7,119.2,123.4,124.6,129.7,130.5,130.9,132.7,135.1,141.9,150.8$, 154.1, 198.2 ppm. MS (EI, 70eV) m/z: $442\left(\mathrm{M}^{+}\right)$. Anal.Calcd for $\mathrm{C}_{20} \mathrm{H}_{16} \mathrm{BrN}_{3} \mathrm{O}_{4}: \mathrm{C}, 54.31 ; \mathrm{H}, 3.65 ; \mathrm{N}$, 9.50; Found: C, 54.20; H, 3.55; N, 9.38\%.

\section{0b-hydroxy-4-nitro-5-(4-nitrophenyl)-2,3,5,5a-tetrahydro-1 $H$-imidazo[1,2-a]indeno[2,1-}

e]pyridin-6(10bH)-one (5h): White solid, MP: 206-207 C, IR (KBr): 3342 (NH), 3195 (OH), 2909, $1712(\mathrm{C}=\mathrm{O}), 1594 \mathrm{~cm}^{-1} ;{ }^{1} \mathrm{H}$ NMR $(300 \mathrm{MHz}, \mathrm{DMSO}-d 6) \delta_{\mathrm{H}}: 3.16-3.25\left(\mathrm{~m}, 1 \mathrm{H}, \mathrm{CH}_{2}\right), 3.50-3.56(\mathrm{~m}$, $\left.1 \mathrm{H}, \mathrm{CH}_{2}\right), 3.62(\mathrm{~s}, 1 \mathrm{H}, \mathrm{CH}), 3.68-3.74\left(\mathrm{~m}, 2 \mathrm{H}, \mathrm{CH}_{2}\right), 3.95-4.04\left(\mathrm{~m}, 1 \mathrm{H}, \mathrm{CH}_{2}\right), 5.03(\mathrm{~s}, 1 \mathrm{H}, \mathrm{CH}), 7.10(\mathrm{~s}$, 1H, OH), 7.58-766 (m, 3H, Ar-H), 7.77 (d, 1H, J=7.5 Hz, Ar-H), 7.82-7.89 (m, 2H, Ar-H), 8.16 (d, 2H, $J=8.7 \mathrm{~Hz}, \mathrm{Ar}-\mathrm{H}), 9.13(\mathrm{~s}, 1 \mathrm{H}, \mathrm{NH}) \mathrm{ppm} ;{ }^{13} \mathrm{C}$ NMR $(75 \mathrm{MHz}, \mathrm{DMSO}-d 6) \delta_{\mathrm{c}}: 36.8,42.3,43.5,63.0$, $85.5,103.2,123.5,123.6,124.8,129.0,130.6,133.0,135.2,146.2,150.9,151.1,154.1,197.8$ ppm. MS (EI, 70eV) m/z: $408\left(\mathrm{M}^{+}\right)$. Anal.Calcd for $\mathrm{C}_{20} \mathrm{H}_{16} \mathrm{~N}_{4} \mathrm{O}_{6}: \mathrm{C}, 58.82 ; \mathrm{H}, 3.95 ; \mathrm{N}, 13.72 \%$. Found: C, 58.71; $\mathrm{H}, 3.83 ; \mathrm{N}, 13.62 \%$

10b-hydroxy-5-(4-methoxyphenyl)-4-nitro-2,3,5,5a-tetrahydro-1H-imidazo[1,2-a]indeno[2,1e]pyridin-6(10bH)-one (5i): White solid, MP: 210-212 ${ }^{\circ} \mathrm{C}$, IR (KBr): 3295 (NH), $3176(\mathrm{OH}), 2960$, $1720(\mathrm{C}=\mathrm{O}), 1588 \mathrm{~cm}^{-1} ;{ }^{1} \mathrm{H}$ NMR (300 MHz, DMSO-d6) $\delta_{\mathrm{H}}: 3.19-3.28\left(\mathrm{~m}, 1 \mathrm{H}, \mathrm{CH}_{2}\right), 3.49-3.55(\mathrm{~m}$, 
2H, $\left.\mathrm{CH}_{2}, \mathrm{CH}\right), 3.63-3.69$ (m, 1H, $\left.\mathrm{CH}_{2}\right), 3.73\left(\mathrm{~s}, 3 \mathrm{H}, \mathrm{CH}_{3}\right), 3.95-4.04\left(\mathrm{~m}, 1 \mathrm{H}, \mathrm{CH}_{2}\right), 4.92(\mathrm{~s}, 1 \mathrm{H}, \mathrm{CH})$, $6.75(\mathrm{~d}, 1 \mathrm{H}, J=7.5 \mathrm{~Hz}, \mathrm{Ar}-\mathrm{H}), 6.88(\mathrm{~m}, 2 \mathrm{H}, \mathrm{Ar}-\mathrm{H}), 6.97(\mathrm{~s} .1 \mathrm{H}, \mathrm{OH}), 7.20(\mathrm{t}, 1 \mathrm{H}, J=8.1 \mathrm{~Hz}, \mathrm{Ar}-\mathrm{H}), 7.61-$ 7.65 (m, 1H, Ar-H), 7.75 (d, 1H, J=7.8 Hz, Ar-H), 7.81-7.88 (m, 2H, Ar-H), 9.21 (s, 1H, NH) ppm; ${ }^{13} \mathrm{C}$ NMR (75 MHz, DMSO-d6) $\delta_{c}: 36.6,42.1,43.3$, 54.8, 63.3, 85.6, 103.9, 111.2, 113.6, 119.8, 123.4, 124.7, 129.0, 130.4, 132.9, 135.0, 144.3, 151.1, 154.1, 159.1, 198.4 ppm. MS (EI, 70eV) m/z: 393 $\left(\mathrm{M}^{+}\right)$. Anal.Calcd for $\mathrm{C}_{21} \mathrm{H}_{19} \mathrm{~N}_{3} \mathrm{O} 5: \mathrm{C}, 64.12 ; \mathrm{H}, 4.87 ; \mathrm{N}, 10.68 \%$. Found: C, 64.01; H, 4.75; N, $10.56 \%$.

\section{ACKNOWLEDGMENTS}

We gratefully acknowledge financial support from the Research Council of Iran National Science Foundation: INSF.

Table 2: Smiles code of the synthesized compounds 5a-i

\begin{tabular}{cl}
\hline Compound & Smiles code \\
\hline $\mathbf{5 a}$ & $\mathrm{c} 12 \mathrm{c}(\mathrm{cccc} 1) \mathrm{C}(=\mathrm{O})[\mathrm{C} @ @ \mathrm{H}] 1[\mathrm{C} @ @] 2(\mathrm{O}) \mathrm{N} 2 \mathrm{CCNC} 2=\mathrm{C}([\mathrm{C} @ \mathrm{H}] 1 \mathrm{c} 1 \mathrm{ccccc} 1) \mathrm{N}(=\mathrm{O})=\mathrm{O}$ \\
$\mathbf{5 b}$ & $\mathrm{c} 12 \mathrm{c}(\mathrm{cccc} 1) \mathrm{C}(=\mathrm{O})[\mathrm{C} @ @ \mathrm{H}] 1[\mathrm{C} @ @] 2(\mathrm{O}) \mathrm{N} 2 \mathrm{CCNC} 2=\mathrm{C}([\mathrm{C} @ \mathrm{H}] 1 \mathrm{c} 1 \mathrm{ccc}(\mathrm{cc} 1) \mathrm{C}) \mathrm{N}(=\mathrm{O})=\mathrm{O}$ \\
$\mathbf{5 c}$ & $\mathrm{c} 12 \mathrm{c}(\mathrm{cccc} 1) \mathrm{C}(=\mathrm{O})[\mathrm{C} @ @ \mathrm{H}] 1[\mathrm{C} @ @] 2(\mathrm{O}) \mathrm{N} 2 \mathrm{CCNC} 2=\mathrm{C}([\mathrm{C} @ \mathrm{H}] 1 \mathrm{c} 1 \mathrm{cnccc} 1) \mathrm{N}(=\mathrm{O})=\mathrm{O}$ \\
$\mathbf{5 d}$ & $\mathrm{c} 12 \mathrm{c}(\mathrm{cccc} 1) \mathrm{C}(=\mathrm{O})[\mathrm{C} @ @ \mathrm{H}] 1[\mathrm{C} @ @] 2(\mathrm{O}) \mathrm{N} 2 \mathrm{CCNC}=\mathrm{C}([\mathrm{C} @ \mathrm{H}] 1 \mathrm{c} 1 \mathrm{cccc}(\mathrm{c} 1) \mathrm{Cl}) \mathrm{N}(=\mathrm{O})=\mathrm{O}$ \\
$\mathbf{5 e}$ & $\mathrm{c} 12 \mathrm{c}(\mathrm{cccc} 1) \mathrm{C}(=\mathrm{O})[\mathrm{C} @ @ \mathrm{H}] 1[\mathrm{C} @ @] 2(\mathrm{O}) \mathrm{N} 2 \mathrm{CCNC}=\mathrm{C}([\mathrm{C} @ \mathrm{H}] 1 \mathrm{c} 1 \mathrm{ccccc} 1 \mathrm{~N}(=\mathrm{O})=\mathrm{O}) \mathrm{N}(=\mathrm{O})=\mathrm{O}$ \\
$\mathbf{5 f}$ & $\mathrm{c} 12 \mathrm{c}(\mathrm{cccc} 1) \mathrm{C}(=\mathrm{O})[\mathrm{C} @ @ \mathrm{H}] 1[\mathrm{C} @ @] 2(\mathrm{O}) \mathrm{N} 2 \mathrm{CCNC} 2=\mathrm{C}([\mathrm{C} @ \mathrm{H}] 1 \mathrm{c} 1 \mathrm{cccc}(\mathrm{c} 1) \mathrm{N}(=\mathrm{O})=\mathrm{O}) \mathrm{N}(=\mathrm{O})=\mathrm{O}$ \\
$\mathbf{5 g}$ & $\mathrm{c} 12 \mathrm{c}(\mathrm{cccc} 1) \mathrm{C}(=\mathrm{O})[\mathrm{C} @ @ \mathrm{H}] 1[\mathrm{C} @ @] 2(\mathrm{O}) \mathrm{N} 2 \mathrm{CCNC}=\mathrm{C}([\mathrm{C} @ \mathrm{H}] 1 \mathrm{c} 1 \mathrm{ccc}(\mathrm{cc} 1) \mathrm{Br}) \mathrm{N}(=\mathrm{O})=\mathrm{O}$ \\
$\mathbf{5 h}$ & $\mathrm{c} 12 \mathrm{c}(\mathrm{cccc} 1) \mathrm{C}(=\mathrm{O})[\mathrm{C} @ @ \mathrm{H}] 1[\mathrm{C} @ @] 2(\mathrm{O}) \mathrm{N} 2 \mathrm{CCNC} 2=\mathrm{C}([\mathrm{C} @ \mathrm{H}] 1 \mathrm{c} 1 \mathrm{ccc}(\mathrm{cc} 1) \mathrm{N}(=\mathrm{O})=\mathrm{O}) \mathrm{N}(=\mathrm{O})=\mathrm{O}$ \\
$\mathbf{5 i}$ & $\mathrm{c} 12 \mathrm{c}(\mathrm{cccc} 1) \mathrm{C}(=\mathrm{O})[\mathrm{C} @ @ \mathrm{H}] 1[\mathrm{C} @ @] 2(\mathrm{O}) \mathrm{N} 2 \mathrm{CCNC}=\mathrm{C}([\mathrm{C} @ \mathrm{H}] 1 \mathrm{c} 1 \mathrm{ccc}(\mathrm{c} 1) \mathrm{OC}) \mathrm{N}(=\mathrm{O})=\mathrm{O}$ \\
\hline
\end{tabular}


Fig 3. A) Crystal structure of HIV protease enzyme. (B) Crystal structure of HIV protease enzyme in complex with Saquinavir from Protein Data Bank. (C) Saquinavir was retrieved from Pubchem substance database

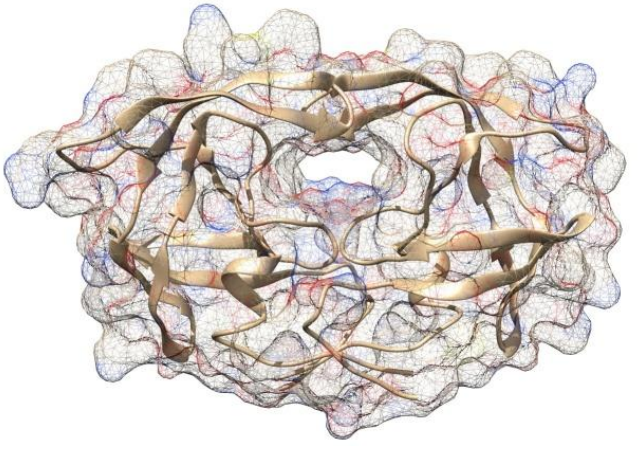

(A)

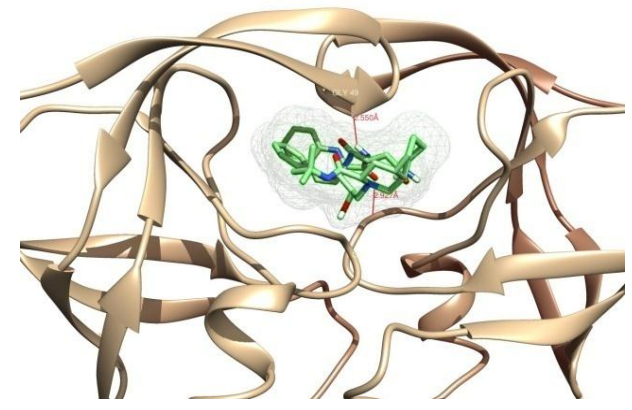

(B)<smiles>CC(C)(C)NC(=O)[C@@H]1C[C@H]2CCCC[C@H]2CN1C[C@H](O)[C@H](Cc1ccccc1)NC(=O)[C@H](CC(N)=O)NC(=O)c1ccc2ccccc2n1</smiles>

(C)

Fig 4. Binding Site of HIV-Protease with all new synthesized compounds and saquinavir. (Autodock 4)

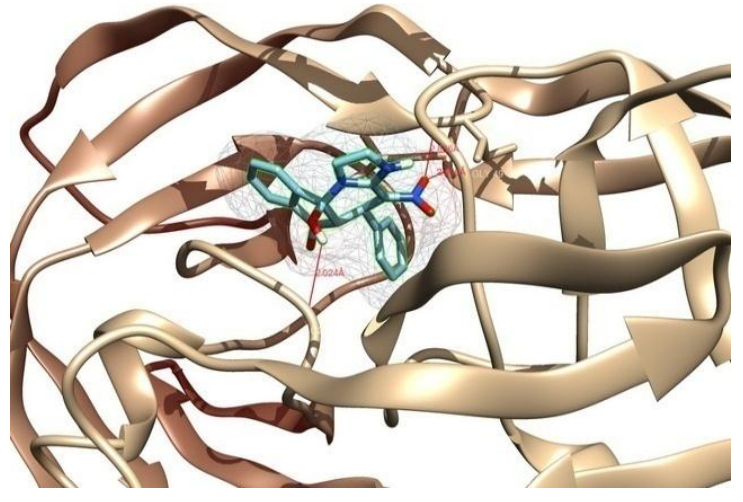

5a

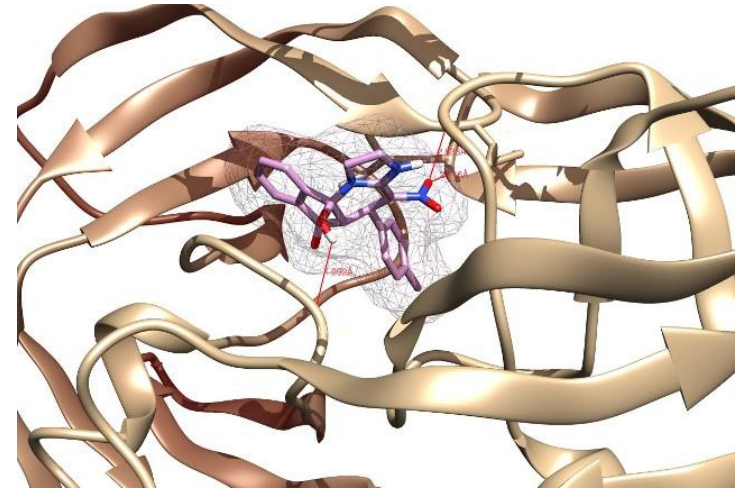

5b 

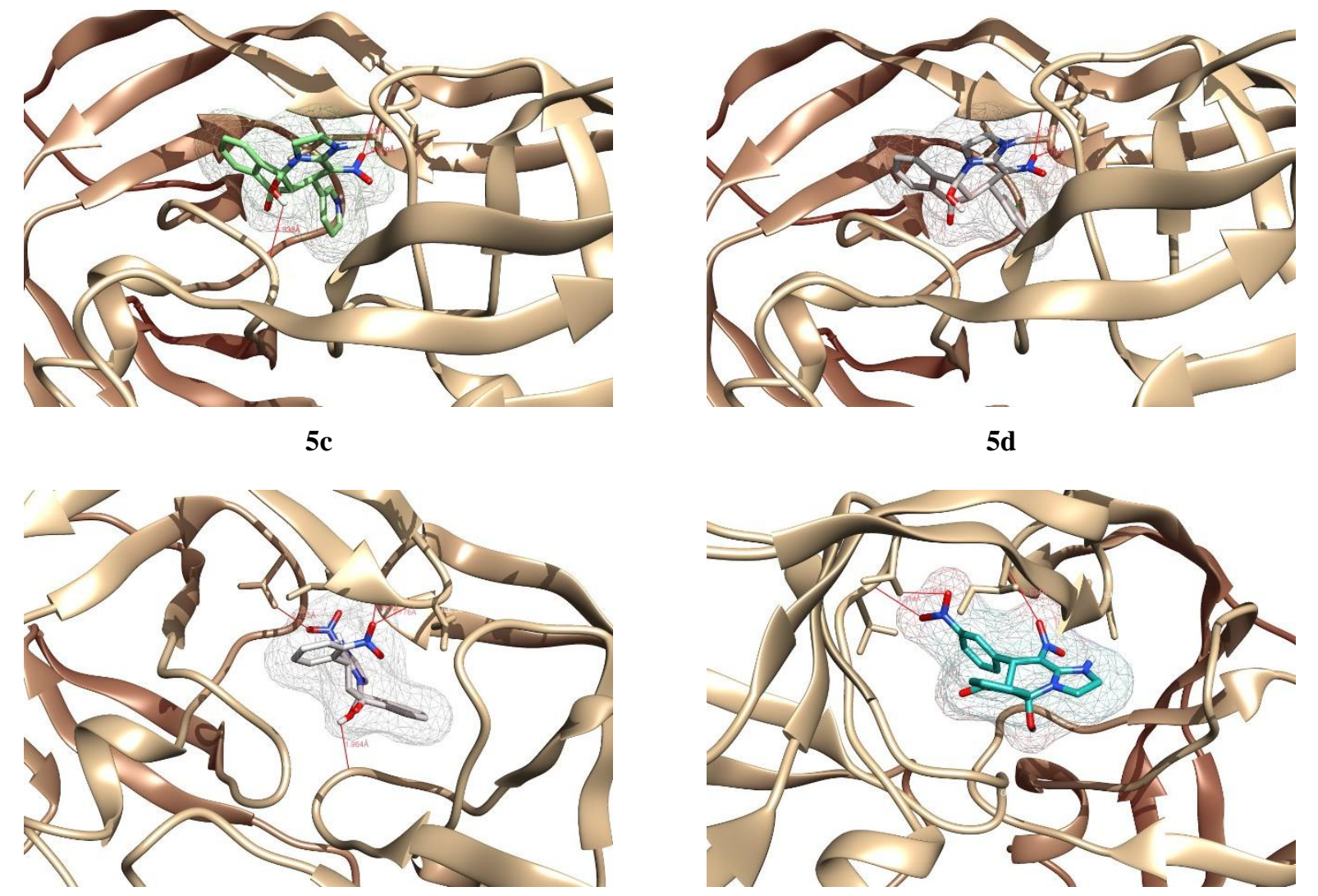

5 e
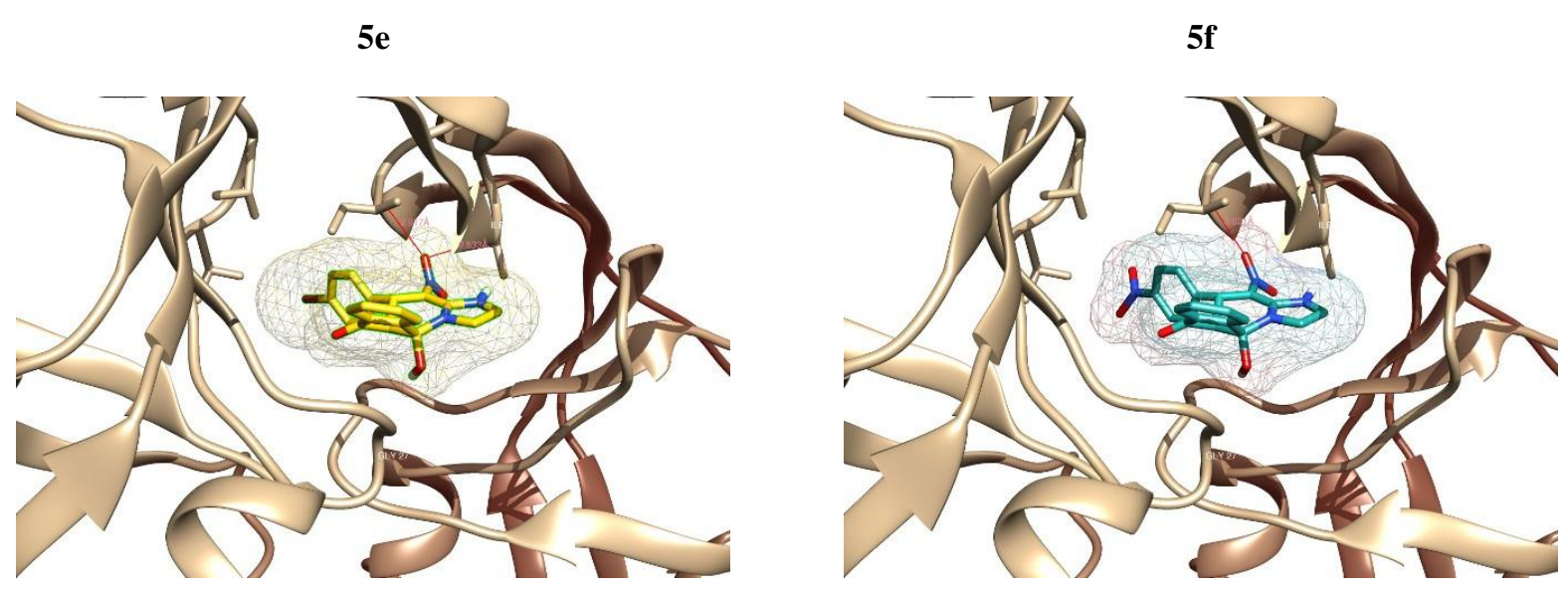

$5 g$

$5 h$

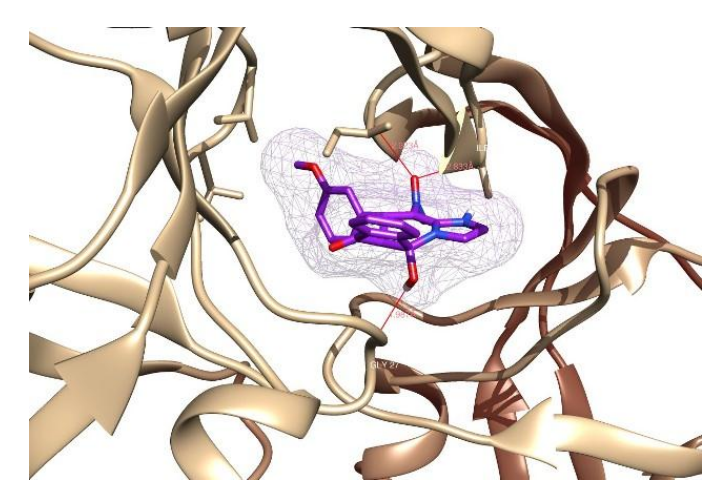

$5 \mathbf{i}$

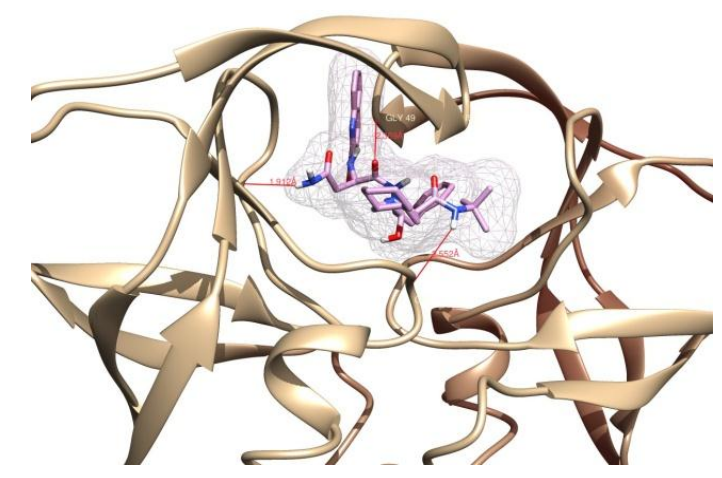

Saquinavir 
Fig 5. Docking of the hydrogen bonding amino acids of receptor HIV protease enzyme with newly synthesized and Saquinavir in the active site of target protein.

Binding Site of HIV-Protease (Autodock 4)

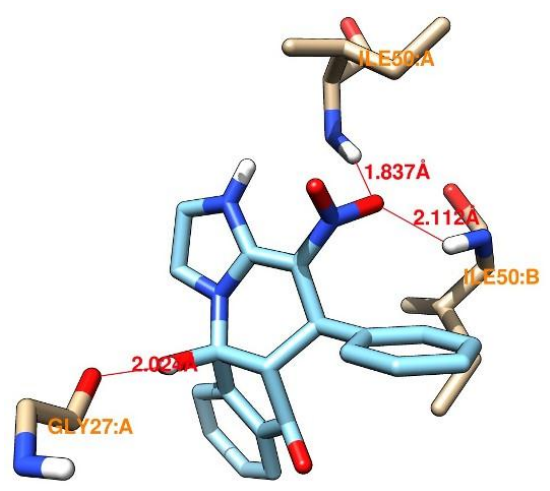

5a:GLY27, ILE50:A, ILE50:B

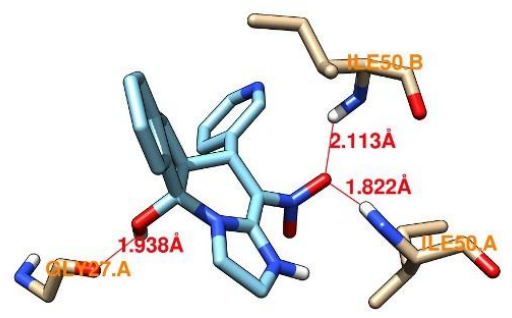

5c: GLY27, ILE50.A, ILE50.B

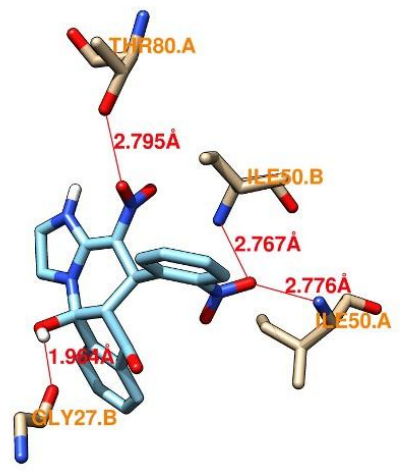

5e: GLY27, ILE50.A, ILE50.B, THR80.A

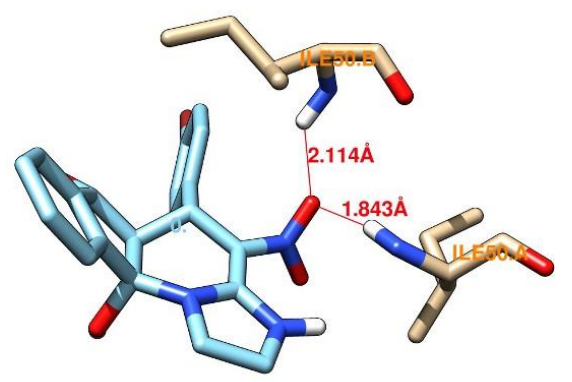

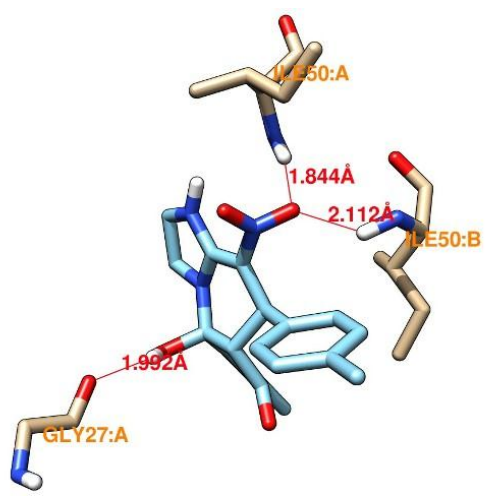

5b: GLY27, ILE50.A, ILE50.B

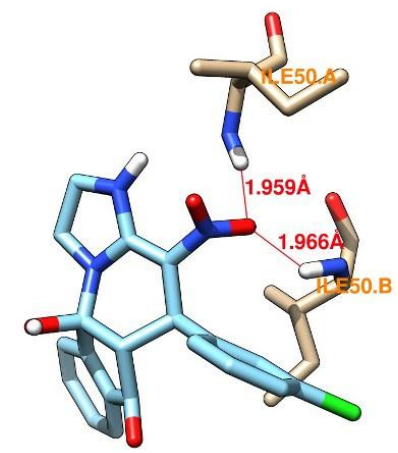

5d: ILE50.A, ILE50.B

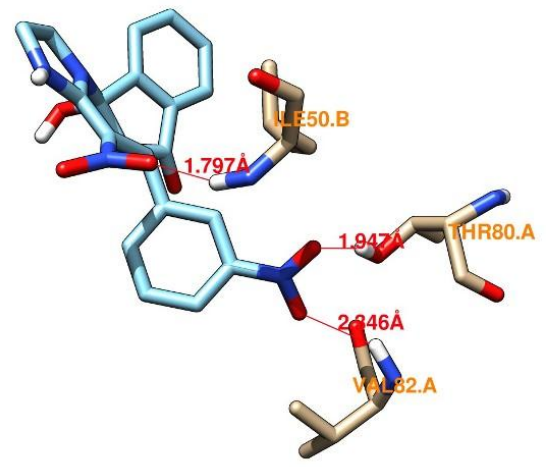

5f: ILE50.B, THR80.A, VAL32.A

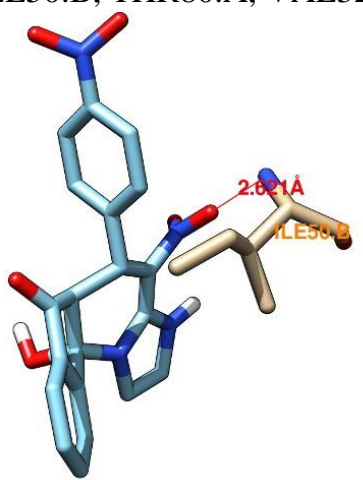

5h:ILE50.B

5g: ILE50.A, ILE50.B 


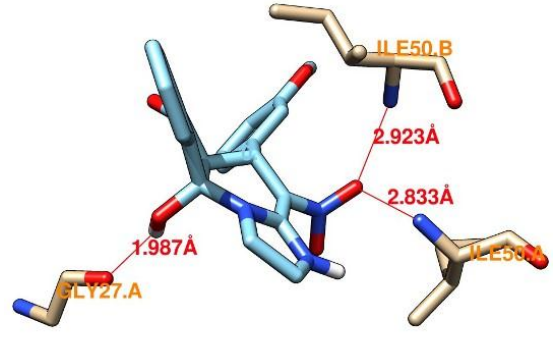

5i: GLY27, ILE50:A, ILE50:B

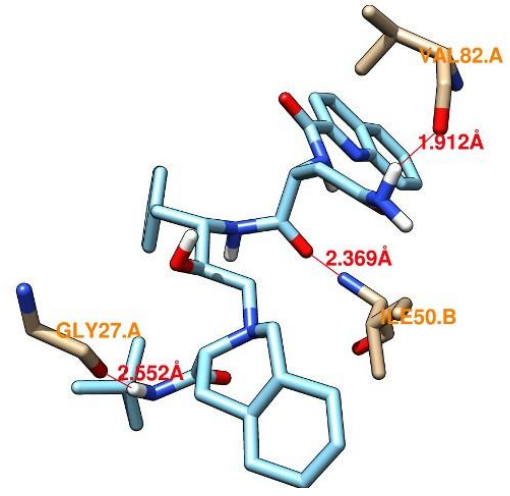

Saquinavir_Pubchem:GLY27.A, ILE50.B, VAL82.A

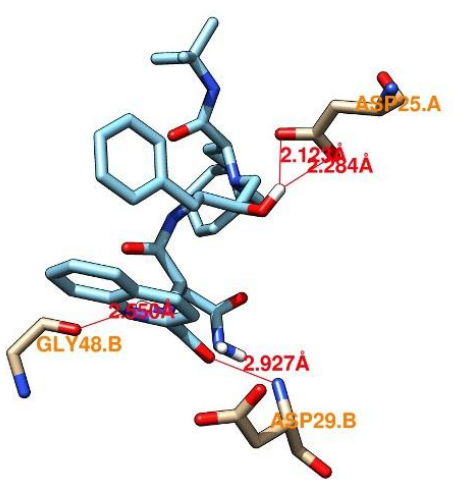

Saquinavir_Crystal:GLY48.B, ASP25.A, ASP29.B

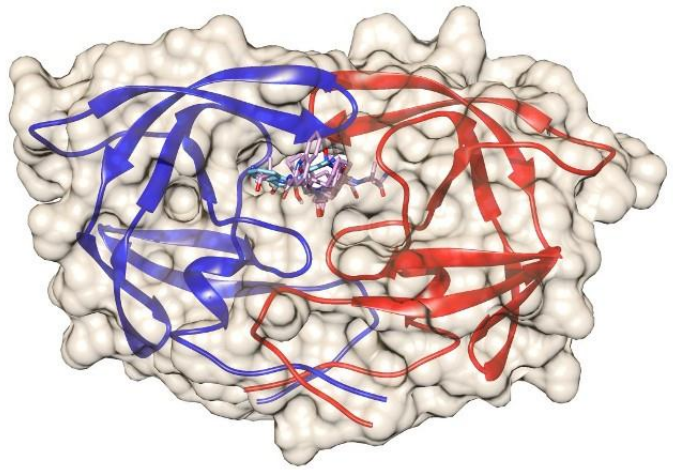

Crystal of HIV-Protease: Chain A\&B

Table 3: Binding energy and Inhibition Constant of the 10b-hydroxy-4-nitro-5-phenyl-2,3,5,5a tetrahydro- $1 H$-imidazo[1,2-a]indeno[2,1-e]pyridin-6(10bH)-one $(5 \mathrm{a}-\mathrm{i})$ and the standard drug Saquinavir.

\begin{tabular}{|c|c|c|c|c|c|c|c|}
\hline \multirow[b]{2}{*}{ Entry } & \multirow[t]{2}{*}{ Ligand } & \multicolumn{4}{|c|}{$\begin{array}{c}\text { Free Energy of } \\
\text { Binding }(\mathrm{kcal} / \mathrm{mol})\end{array}$} & \multirow[t]{2}{*}{$\begin{array}{l}\text { Estimated Inhibition } \\
\text { Constant, } \mathrm{Ki}\end{array}$} & \multirow[t]{2}{*}{$\begin{array}{l}\text { Hydrophobic } \\
\text { interactions }\end{array}$} \\
\hline & & \multicolumn{2}{|c|}{ Auto Dock 4} & \multicolumn{2}{|c|}{ Auto Dock Vina } & & \\
\hline 1 & Saquinavire_Crystal ${ }^{*}$ & \multicolumn{2}{|c|}{-12.20} & \multicolumn{2}{|c|}{-10.7} & $1.15 \mathrm{nM}$ (nanomolar) & \\
\hline 2 & Saquinavire_Pubchem ${ }^{* *}$ & \multicolumn{2}{|c|}{-12.63} & \multicolumn{2}{|c|}{-9.7} & $1.15 \mathrm{nM}$ (nanomolar) & \\
\hline 3 & Structure & 5 & $\begin{array}{l}\text { mirror } \\
\text { image }\end{array}$ & 5 & $\begin{array}{l}\text { mirror } \\
\text { image }\end{array}$ & & \\
\hline 4 & Structure 5a & -8.01 & -7.83 & -9.9 & -8.4 & $1.35 \mathrm{uM}$ (micromolar) & \\
\hline 5 & Structure $5 b$ & -8.28 & -7.85 & -9.4 & -9.8 & $\begin{array}{l}847.06 \mathrm{nM} \\
\text { (nanomolar) }\end{array}$ & \\
\hline 6 & Structure $5 \mathrm{c}$ & -7.73 & -7.58 & -9.2 & -9.0 & $2.17 \mathrm{uM}$ (micromolar) & \\
\hline 7 & Structure $5 d$ & -8.75 & -7.71 & -9.3 & -8.9 & $\begin{array}{l}387.35 \mathrm{nM} \\
\text { (nanomolar) }\end{array}$ & 安 \\
\hline 8 & Structure $5 \mathrm{e}$ & -8.22 & -7.66 & -9.0 & -8.0 & $\begin{array}{l}941.20 \mathrm{nM} \\
\text { (nanomolar) }\end{array}$ & \\
\hline 9 & Structure $5 \mathrm{f}$ & -9.24 & -7.58 & -9.1 & -8.9 & $\begin{array}{l}214.82 \mathrm{nM} \\
\text { (nanomolar) }\end{array}$ & \\
\hline 10 & Structure $5 \mathrm{~g}$ & -8.45 & -8.23 & -9.2 & -9.4 & $\begin{array}{l}645.23 \mathrm{nM} \\
\text { (nanomolar) }\end{array}$ & \\
\hline 11 & Structure $5 \mathrm{~h}$ & -7.69 & -8.22 & -9.3 & -8.3 & $2.29 \mathrm{uM}$ (micromolar) & \\
\hline 12 & Structure $5 \mathrm{i}$ & -8.36 & -7.90 & -8.8 & -8.6 & $\begin{array}{l}744.18 \mathrm{nM} \\
\text { (nanomolar) }\end{array}$ & \\
\hline
\end{tabular}




\section{References}

[1] P.A. Wender, J.M. Schaus, A.W. White, General methodology for cis-hydroisoquinoline synthesis: synthesis of reserpine, J. Am. Chem. Soc. 102(19) (1980) 6157-6159.

[2] M.T. Reding, T. Fukuyama, Stereocontrolled Total Synthesis of ( \pm )-Catharanthine via Radical-Mediated Indole Formation, Org. Lett. 1(7) (1999) 973-976.

[3] G. Barbe, A.B. Charette, Total Synthesis of (+)-Lepadin B: Stereoselective Synthesis of Nonracemic Polysubstituted Hydroquinolines Using an RC-ROM Process, J. Am. Chem. Soc. 130(42) (2008) 13873-13875.

[4] V. N'Goka, G. Schlewer, J.M. Linget, J.P. Chambon, C.G. Wermuth, GABA-uptake inhibitors: construction of a general pharmacophore model and successful prediction of a new representative, J. Med. Chem. 34(8) (1991) 2547-2557.

[5] G.A.R. Johnston, P. Krogsgaard-Larsen, A.L. Stephanwn, B. Twitchin, INHIBITION OF THE UPTAKE OF GABA AND RELATED AMINO ACIDS IN RAT BRAIN SLICES BY THE OPTICAL ISOMERS OF NIPECOTIC ACID, J. Neurochem. 26(5) (1976) 1029-1032.

[6] L. Garrido, E. Zubía, M.J. Ortega, J. Salvá, Haouamines A and B: A New Class of Alkaloids from the Ascidian Aplidium haouarianum, J. Org. Chem. 68(2) (2003) 293-299.

[7] P.L. Katavic, D.A. Venables, P.I. Forster, G. Guymer, A.R. Carroll, Grandisines C-G, Indolizidine Alkaloids from the Australian Rainforest Tree Elaeocarpus grandis, J. Nat. Prod. 69(9) (2006) 1295-1299.

[8] Q. Liu, Y.-A. Zhang, P. Xu, Y. Jia, Total Synthesis of (+)-Lysergic Acid, J. Org. Chem. 78(21) (2013) 10885-10893. [9] M.A. Chowdhury, K.R.A. Abdellatif, Y. Dong, E.E. Knaus, Synthesis of new 4-[2-(4-methyl(amino)sulfonylphenyl)-5trifluoromethyl-2H-pyrazol-3-yl]-1,2,3,6-tetrahydropyridines: A search for novel nitric oxide donor anti-inflammatory agents, Bioorg. Med. Chem. 16(19) (2008) 8882-8888.

[10] S.K. Raju, M.R. Stephen, P. Subbu, B. Debjani, Y. Perumal, S. Dharmarajan, A Facile Synthesis and Discovery of Highly Functionalized Tetrahydropyridines

and Pyridines as Antimycobacterial Agents, Chem. Pharm. Bull. 58(5) (2010) 602-610.

[11] M. Misra, S.K. Pandey, V.P. Pandey, J. Pandey, R. Tripathi, R.P. Tripathi, Organocatalyzed highly atom economic one pot synthesis of tetrahydropyridines as antimalarials, Bioorg. Med. Chem. 17(2) (2009) 625-633.

[12] J.P. Cueva, A. Gallardo-Godoy, J.I. Juncosa, P.A. Vidi, M.A. Lill, V.J. Watts, D.E. Nichols, Probing the Steric Space

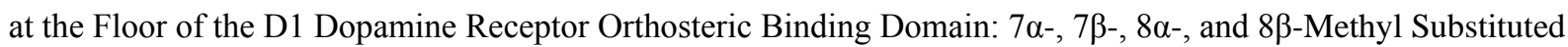
Dihydrexidine Analogues, J. Med. Chem. 54(15) (2011) 5508-5521. 
[13] P.R. Krishna, P.S. Reddy, "Diversity Oriented Synthesis” of Functionalized Chiral Tetrahydropyridines: Potential GABA Receptor Agonists and Azasugars from Natural Amino Acids via a Sequential Baylis-Hillman Reaction and RCM Protocol, J. Comb. Chem. 10(3) (2008) 426-435.

[14] J.D. Scott, S.W. Li, H. Wang, Y. Xia, C.L. Jayne, M.W. Miller, R.A. Duffy, G.C. Boykow, T.J. Kowalski, B.D. Spar, A.W. Stamford, S. Chackalamannil, J.E. Lachowicz, W.J. Greenlee, Diaryl piperidines as CB1 receptor antagonists, Bioorg. Med. Chem. Lett. 20(3) (2010) 1278-1283.

[15] W. Zhang, Y. Chen, W. Chen, Z. Liu, Z. Li, Designing Tetrahydroimidazo[1,2-a]pyridine Derivatives via Catalyst-free Aza-Diels-Alder Reaction (ADAR) and Their Insecticidal Evaluation, J. Agric. Food. Chem. 58(10) (2010) 6296-6299.

[16] S. Sun, C. Cheng, J. Yang, A. Taheri, D. Jiang, B. Zhang, Y. Gu, Synthesis of Tetrahydropyridine Derivatives through a Modular Assembly Reaction Using 3,4-Dihydropyran as Dual Substrate and Template, Org. Lett. 16(17) (2014) 45204523.

[17] J.-P. Wan, Y. Lin, Q. Huang, Y. Liu, Diastereoselective Construction of Tetrahydropyridine Fused Bicyclic Structures via Three-Component Domino Reaction, J. Org. Chem. 79(15) (2014) 7232-7238.

[18] M. Yıldırım, D. Çelikel, Y. Dürüst, D.W. Knight, B.M. Kariuki, A rapid and efficient protocol for the synthesis of novel nitrothiazolo[3,2-c]pyrimidines via microwave-mediated Mannich cyclisation, Tetrahedron 70(12) (2014) $2122-2128$. [19] A.A. Mohammadi, S. Askari, H. Rohi, A.A. Soorki, Design, Synthesis, and Antibacterial Evaluation of Some Novel 3'(Phenylamino)-1'H-spiro[indoline-3,2'-quinazoline]-2,4'(3'H)-dione Derivatives, Synth. Commun. 44(4) (2014) $457-467$.

[20] A.A. Mohammadi, R. Akbarzadeh, H. Rouhi, Multicomponent one-pot reactions: Synthesis of some new 6-oxopyrano [2,3-c]isochromenes by condensation of homophthalic anhydride, dialkyl acetylenedicarboxylate, and isocyanides, Comb. Chem. High Throughput Screen. 12(5) (2009) 536-542.

[21] A.A. Mohammadi, M. Mivechi, H. Kefayati, Potassium aluminum sulfate (alum): An efficient catalyst for the one-pot synthesis of trisubstituted imidazoles, Monatsh. Chem. 139(8) (2008) 935-937.

[22] A.A. Mohammadi, S. Taheri, S. Askari, R. Ahdenov, KAl(SO4)2.12H2O(Alum): An Efficient Catalyst for the Synthesis of Novel bis[spiro(quinazoline-oxindole)] Derivatives Via One-Pot Pseudo Five-Component Reactions, J. Heterocycl. Chem. 52(6) (2015) 1871-1875.

[23] J. Azizian, A.A. Mohammadi, A.E. Karimi, M.R. Mohammadizadeh, A stereoselective three-component reaction: $\mathrm{KAl}(\mathrm{SO} 4) 2 \cdot 12 \mathrm{H} \mathrm{2O}$, an efficient and reusable catalyst for the one-pot synthesis of cis-isoquinolonic acids, J. Org. Chem. $70(1)(2005)$ 350-352.

[24] A.A. Mohammadi, M. Dabiri, H. Qaraat, A regioselective three-component reaction for synthesis of novel 1'Hspiro[isoindoline-1,2'-quinazoline]-3,4'(3'H)-dione derivatives, Tetrahedron 65(18) (2009) 3804-3808. 
[25] A.A. Mohammadi, S. Tahery, S. Askari, One-pot five-component reaction for synthesis of some novel bisdihydroquinazolinone derivatives, Arkivoc (v) (2014) 310-318.

[26] M. Karplus, Vicinal Proton Coupling in Nuclear Magnetic Resonance, J. Am. Chem. Soc. 85(18) (1963) $2870-2871$. [27] E.a.C.C.H.N.O. Crystal structure of 5e: Crystalized from a 1:1 mixture of THF, monoclinic, space group P 21/n, cell length: $a=9.3575(19), b=15.267(3)$, c $6.460(3)$, beta: 102.11(3),cell_volume $V=2299.2(8) \AA 3$, Z=4, crystal dimensions: $0.20 \times 0.10 \times 0.08 \mathrm{~mm}$, STOE IPDS 2T diffractometer, Mo radiation, $293 \mathrm{~K}$, measurement range: $3.6<2 \theta<50,4052$ independent reflections, programs WinGX-2013.3 and SHELXL-98, 320 parameters, R1 =0.045 and wR2 (all data) =0.093, max/min residual electron density: 0.135/-0.148 eA-3. CCDC-XXXX contains the supplementary crystallographic data for this paper. These data can be obtained free of charge from The Cambridge Crystallographic Data Centre via www.ccdc.cam.ac.uk/data_request/cif.

[28] O. Trott, A.J. Olson, AutoDock Vina: Improving the speed and accuracy of docking with a new scoring function, efficient optimization, and multithreading, J. Comput. Chem. 31(2) (2010) 455-461.

[29] E. Akaho, G. Morris, D. Goodsell, D. Wong, A. Olson, A Study on Docking Mode of HIV Protease and Their Inhibitors, J. Chem. Software, 7(3) (2001) 103-114.

[30] D.J. Kucera, R.W. Scott, HIV protease inhibitors, compositions containing the same, their pharmaceutical uses and materials for their synthesis, US7094909, US, 2006.

[31] A. Krohn, S. Redshaw, J.C. Ritchie, B.J. Graves, M.H. Hatada, Novel binding mode of highly potent HIV-proteinase inhibitors incorporating the (R)-hydroxyethylamine isostere, J. Med. Chem. 34(11) (1991) 3340-3342. 